\title{
Control Group
}

National Cancer Institute

\section{Source}

National Cancer Institute. Control Group. NCI Thesaurus. Code C28143.

A group used as a standard of comparison in a control experiment. 